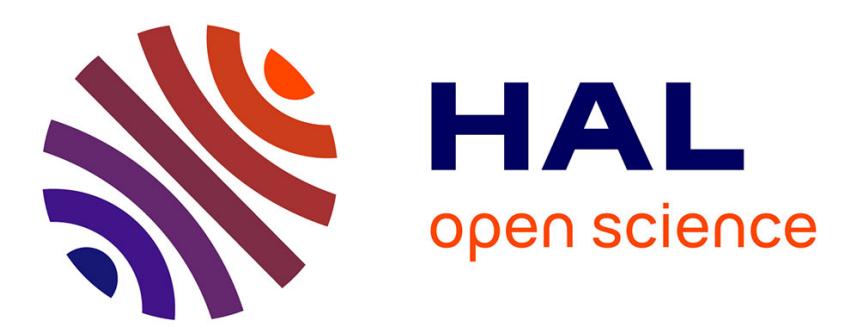

\title{
Efficient Bragg diffraction in thin semiconductor two-dimensional gratings
}

Qiong He, Isabelle Zaquine, Robert Frey, R. André, Gérald Roosen

\section{To cite this version:}

Qiong He, Isabelle Zaquine, Robert Frey, R. André, Gérald Roosen. Efficient Bragg diffraction in thin semiconductor two-dimensional gratings. Optics Letters, 2008, 33 (23), pp.2868-2870. 10.1364/OL.33.002868 . hal-00553478

\section{HAL Id: hal-00553478 \\ https://hal-iogs.archives-ouvertes.fr/hal-00553478}

Submitted on 27 Feb 2012

HAL is a multi-disciplinary open access archive for the deposit and dissemination of scientific research documents, whether they are published or not. The documents may come from teaching and research institutions in France or abroad, or from public or private research centers.
L'archive ouverte pluridisciplinaire HAL, est destinée au dépôt et à la diffusion de documents scientifiques de niveau recherche, publiés ou non, émanant des établissements d'enseignement et de recherche français ou étrangers, des laboratoires publics ou privés. 


\title{
Efficient Bragg diffraction in thin semiconductor two-dimensional gratings
}

\author{
Q. He, ${ }^{1,3}$ I. Zaquine, ${ }^{1, *}$ R. André, ${ }^{2}$ G. Roosen, ${ }^{3}$ and R. Frey ${ }^{1,3}$ \\ ${ }^{1}$ Laboratoire Traitement et Communication de l'Information, CNRS et Institut Télécom, Télécom-ParisTech, \\ dép. TSI, 46 rue Barrault, 75634 Paris Cedex 13, France \\ ${ }^{2}$ CEA-CNRS Group Nanophysique et semiconducteurs, Institut NEEL-CNRS, BP 166, 38042 Grenoble Cedex, France \\ ${ }^{3}$ Laboratoire Charles Fabry de l'Institut d'Optique, CNRS Campus Polytechnique, RD. 128, 91128 Palaiseau \\ Cedex, France \\ *Corresponding author: zaquine@telecom-paristech.fr
}

Received July 28, 2008; accepted October 13, 2008; posted October 30, 2008 (Doc. ID 99403); published November 25, 2008

Highly improved diffraction properties are demonstrated in a two-dimensional [2D] grating consisting of a transmission grating optically recorded in a semiconductor one-dimensional photonic crystal (1D-PC). Near unity internal diffraction efficiency, high wavelength selectivity, and Bragg diffraction regime operation are demonstrated when the read beam is set at Bragg incidence on the transmission grating while its wavelength corresponds to the band edge of the $3 \mu \mathrm{m}$ thick 1D-PC. When the $2 \mathrm{D}$ grating is grown on a Bragg mirror, a single diffracted beam is obtained, which makes the device promising for optical signal processing. (C) 2008 Optical Society of America

OCIS codes: $050.0050,190.2055$.

Optical signal processing greatly benefits from the use of transmission diffraction gratings [1]. For the purpose of compactness, thin nonlinear materials such as polymers, liquid crystals, or semiconductor nanostructures are used [2-5]. However, their small thicknesses imply a Raman-Nath diffraction regime with several diffraction orders, which limits the maximum power reached in the desired order. Such a drawback was circumvented by placing the holographic material in an asymmetric cavity [6-8], and the Bragg operation of the device was obtained, even with a thin recording material $[9,10]$. Recently, another solution using two-dimensional (2D) photonic crystals was proposed and demonstrated in photopolymers [11]. However, due to the low refractive index contrast $(\Delta n=0.045)$ that could be reached in such materials, the device performances remained limited. In this Letter we show that the use of semiconductor 2D gratings exhibiting much larger refractive index contrasts in the mean direction of the read and diffracted beams enables Bragg internal diffraction efficiencies of unity together with high wavelength selectivity, even for micrometer thick devices.

As explained in detail in [11], the technique of band-edge-induced Bragg diffraction in 2D gratings is based on the enhancement of light intensity at the band edge wavelength of the one-dimensional (1D) refractive index modulation along the mean propagation direction $\hat{Z}$ of the read and diffracted beams. Indeed, for a transmission grating with a refractive index modulation perpendicular to the $\hat{Z}$ direction, very strong enhancement of the diffraction efficiency is predicted if the read beam propagation direction is set at Bragg incidence on this transmission grating while its wavelength is at the band edge resonance of the one-dimensional photonic crystal (1D-PC). In such a case, both read and -1 diffraction order intensities are enhanced through the local field amplification $[12,13]$. Higher diffraction orders are emitted at different angles and only benefit from the read beam resonance. Their intensities can be negligible, especially in the case of low efficiency transmission gratings. Indeed, three advantages come from using such a 2D grating device: a huge enhancement of the diffraction efficiency (a factor of 10 to 1000), a largely improved wavelength selectivity (a factor of a few hundreds), and a Bragg diffraction regime with only two diffracted beams (one in transmission, the other one in reflexion). A single diffracted beam counterpropagating the read beam can be obtained if the 1D-PC is deposited on a Bragg mirror [14].

Such a device was realized and tested using the experimental setup shown in Fig. 1. The sample S with a total thickness of $2.44 \mu \mathrm{m}$ consisted of a 1D-PC containing 30 pairs of CdMnTe-CdMgTe layers with respective refractive indices of 3.088 and 2.585 and a band edge wavelength around $694 \mathrm{~nm}$, deposited on a Bragg mirror that was totally reflecting at this wavelength [15]. The refractive index transmission grating was recorded in the 1D-PC thanks to the generation of free carriers through two-photon absorption at a wavelength of $820 \mathrm{~nm}$ that is below the optical bandgap of the 1D-PC. The two write beams of equal intensities interfered on the sample after being sym-

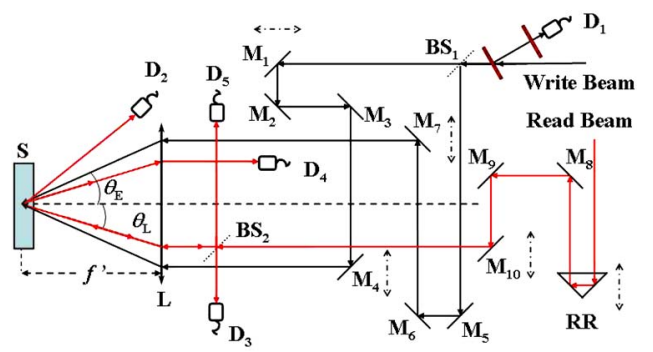

Fig. 1. (Color online) Experimental setup. Detector $\mathrm{D}_{1}$ was used to monitor the pump energy. Detectors $D_{3}$ and $D_{4}$, $D_{3}$ and $D_{5}$, and $D_{3}$ and $D_{2}$ allowed the measurement of the signal reflectivity, -1 order diffraction efficiency, and +1 order diffraction efficiency, respectively. 
metrically focused by a single lens. For a grating period of $4.46 \mu \mathrm{m}$ the write incidence angle $\theta_{W}$ was adjusted to $5.3^{\circ}$ thanks to the moving mirrors $\mathrm{M}_{4}$ and $\mathrm{M}_{7}$, and the synchronization of the two write pulses was ensured by the moving mirror set $\mathrm{M}_{1}-\mathrm{M}_{2}$. The write beams came from one of the two outputs of a dual independently tunable-wavelength optical parametric generator (OPG) pumped by the same frequency-tripled mode locked Nd-YAG laser [16]. The second output of the OPG was tuned around $694 \mathrm{~nm}$ and used as the read beam. This read beam was focused on the sample at the Bragg incidence angle $\theta_{R}=4.5^{\circ}$ of the recorded transmission grating. The Bragg direction was adjusted thanks to the moving mirror $\mathrm{M}_{10}$. The net diffraction efficiency (the measured intensity ratio of the reflected diffracted and incident read beams) was calibrated by replacing the sample by a gold mirror set orthogonally to the read beam.

A spectroscopic study was first undertaken for a write-read delay ( $\Delta t \approx 20 \mathrm{ps}$ ) larger than the pulse duration to maximize the free carrier density generated by the interfering write beams. Figure 2 shows both the read beam reflectivity spectra recorded at a high write fluence $\left(0.62 \mathrm{~mJ} / \mathrm{cm}^{2}\right)$ and in the linear regime with no write beams [Fig. 2(a)] and the net diffraction efficiency spectra of this read beam for the -1 and +1 diffraction orders [Fig. 2(b)]. These spectra were recorded around the band edge wavelength and the hole at $694.1 \mathrm{~nm}$ in the linear reflectivity spectrum was due to diffusion losses at the interfaces of the CdMnTe-CdMgTe layers enhanced by the high local field at the band edge wavelength [13]. At a high write fluence the reflectivity spectrum is blueshifted [15] by $0.27 \mathrm{~nm}$, which corresponds to a mean refractive index change $\Delta n=1.2 \times 10^{-3}$ determined using the procedure described in [17]. For two write beams of equal intensities this gives rise to a refractive index modulation $n_{1}=3 \Delta n_{0} / 2=1.8 \times 10^{-3}$ (free carriers are generated by two-photon absorption), and the diffraction efficiency of the same transmission grating written in a homogeneous medium can be calculated from $\rho_{D \text { hom }}=\left(\pi n_{1} L / \lambda\right)^{2}=1.9 \times 10^{-4}$.

Figure 2(b) shows the huge enhancement of the Bragg ( -1 order) diffraction at the band edge frequency. The diffraction efficiency increases from about $2.5 \times 10^{-4}$ (very close to that estimated for the homogenous medium) far from the band edge resonance to around $1 \%$ at this band edge resonance (an increase by a factor of 40) despite losses introduced

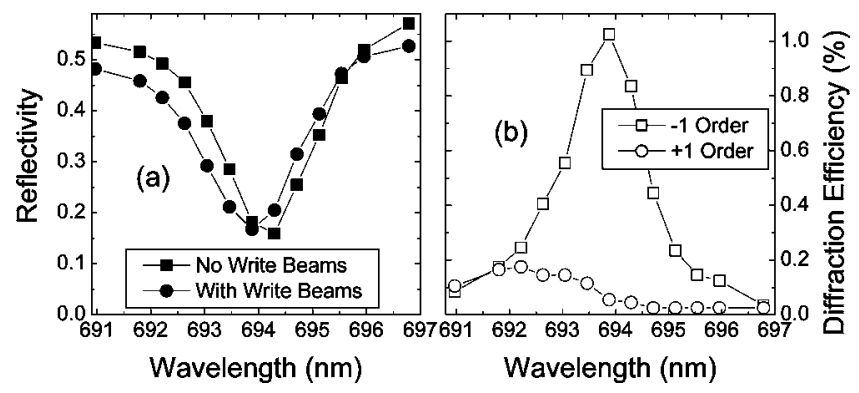

Fig. 2. (a) Reflectivity and (b) diffraction efficiency spectra. by the nanostructuration of the holographic medium. Moreover the device is much more resonant $\left(\Delta \lambda_{\mathrm{CP}}\right.$ $\approx 1.8 \mathrm{~nm}$ ) than the same transmission grating recorded in a homogeneous medium $\left(\Delta \lambda_{\mathrm{CP}}\right.$ $\approx 10,000 \mathrm{~nm}$ calculated for this $1.9 \mu \mathrm{m}$ thick grating). Finally, Fig. 2(b) also represents the net diffraction efficiency spectrum of the +1 diffraction order. It is much less intense than order -1 (a factor of 18 at the band edge) as its diffraction angle is $13.6^{\circ}$. Such behavior demonstrates that a Bragg diffraction regime can be obtained for a very thin 2D grating , which makes this device very attractive for the purpose of optical signal processing.

The maximum net diffraction efficiency obtained at the band edge of the $1 \mathrm{D}-\mathrm{PC}$ was also measured as a function of the total pump fluence. Experimental results are shown in Fig. 3, together with a fit taking into account the two-photon absorption-induced free carrier origin of the refractive index modulation. The net diffraction efficiency $\rho_{\text {net }}$ can then be phenomenologically described by

$$
\rho_{\text {net }}=T_{\mathrm{CP}}^{2} \exp \left(-2 \alpha F_{P}^{2}\right) \sin ^{2}\left(\Delta \Phi F_{P}^{2}\right),
$$

where $1-T_{\mathrm{CP}}$ and $1-\exp \left(-\alpha F_{P}^{2}\right)$ represent optical losses for both read and diffracted beams, respectively, due to the linear diffusion at the CdMnTeCdMgTe interfaces (the value $T_{\mathrm{CP}}=0.17$ was taken from Fig. 2) and the linear absorption due to the free carriers photogenerated through two-photon absorption of the write beams of total fluence $F_{P}\left(\alpha=0.08 \mathrm{~cm}^{4} \mathrm{~mJ}^{-2}\right.$ was determined by the fit); $\rho_{\text {int }}=\sin ^{2}\left(\Delta \Phi F_{P}^{2}\right)$ represents the internal diffraction efficiency of the device at the write fluence $F_{P}\left(\Delta \Phi=0.27 \mathrm{~cm}^{4} \mathrm{~mJ}^{-2}\right.$ was determined by the fit $)$. The excellent quality of the fit is actually a validation of the phenomenological Eq. (1). The fit also demonstrated that the internal conversion efficiency nearly reached $100 \%$ (93\% at the maximum pump fluence used). The small value (1.3\%) of the measured net diffraction efficiency was then only due to losses (a factor of about 35 for the diffusion losses and a factor of 2 for free carrier absorption losses). Considering the possibility of applying such a device to optical signal processing, these large losses indicate that the transmission grating had better be recorded either optically using electronic Kerr effect or, even better, electrically using Pockels effect with a comb electrode. More important, the linear losses should be almost suppressed by increasing the sample quality or (and) using thinner 1D-PCs of a larger refractive index contrast [17].

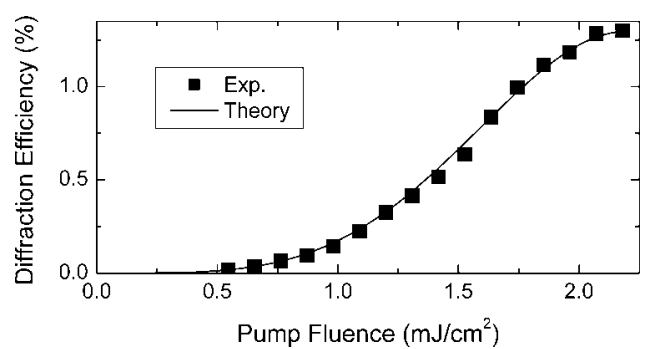

Fig. 3. Diffraction efficiency versus write fluence. 
Time-resolved write-read experiments were also performed to verify the postulated origin of the refractive index modulation. Figure 4 shows the experimental net diffraction efficiency $\rho_{\text {net }}(\delta t)$ of the grating plotted as a function of the write-read delay $\delta t$ for a pump fluence of $1.75 \mathrm{~mJ} \mathrm{~cm}{ }^{2}$ together with a fit performed using the expression [derived from Eq. (1)]

$$
\begin{aligned}
\rho_{\text {net }}(\delta t)= & \frac{T_{\mathrm{CP}}^{2}}{\sqrt{\pi} \Delta t} \int_{-\infty}^{+\infty} \sin ^{2}[\Phi(t)] \exp [-2 \alpha(t)] \\
& \times \exp \left[-\left(\frac{t-\delta t}{\Delta t}\right)^{2}\right] \mathrm{d} t,
\end{aligned}
$$

with $\Phi(t)=\sqrt{2 / \pi}\left(\Phi_{0} / \Delta t\right) \int_{-\infty}^{t} \exp \left[-2 \tau^{2} / \Delta t^{2}\right] \mathrm{d} \tau$ and $\alpha(t)=\sqrt{2 / \pi}\left(\alpha_{0} / \Delta t\right) \int_{-\infty}^{t} \exp \left[-2 \tau^{2} / \Delta t^{2}\right] \mathrm{d} \tau, \quad$ where $\Phi_{0}=0.82$ and $\alpha_{0}=0.23$ were given by the fit of the experimental results shown in Fig. 3 . The pulse duration $\Delta t$ was then the only free parameter in this fit. The very high quality of the fit shown in Fig. 4 for $\Delta t=10 \mathrm{ps}$ is further proof of the validity of Eq. (1). The long plateau observed at large delays shows that carrier diffusion is negligible in the results of Fig. 3, but it could also be detrimental to fast optical processing. From this point of view also, instantaneous electronic Kerr and Pockels effects would evidently be much more interesting for the recording of the transmission gratings.

The near unity internal conversion efficiency was obtained using a linearly polarized read beam. As in our device the diffracted beam is counterpropagating to the read beam; the best effective conversion efficiency (the intensity ratio of the diffracted beam coming out of the device to that of the read laser) is only $25 \%$ (when using a 50-50 beam splitter). As mentioned earlier, this drawback could in principle be circumvented by using a circularly polarized read beam, provided the response of the diffraction grating is isotropic [14]. This proposition was tested by replacing the beam splitter $\mathrm{BS}_{2}$ by a polarizing beam splitter and a quarter-wave plate. The results were exactly the same, but the effective diffraction efficiency was measured rather than the net one. As expected all the diffracted light was collected at the output of the

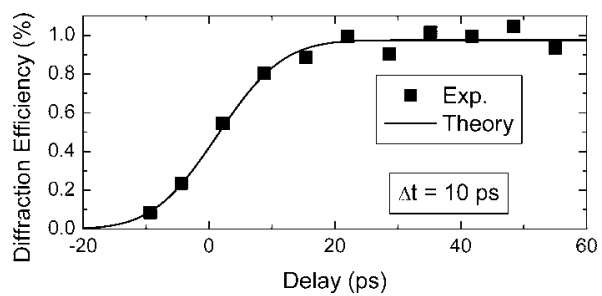

Fig. 4. Diffraction efficiency versus write-read delay. polarizing beam splitter, which is very promising for application to dense optical signal processing.

In conclusion, diffraction of transmission gratings recorded orthogonally to a semiconductor $1 \mathrm{D}-\mathrm{PC}$ was studied at the band edge wavelength of the 1D-PC. For this very thin 2D grating, an internal diffraction efficiency close to unity was measured for the transmission grating with an increase by a factor of 4,000 when compared to the same grating recorded in a homogeneous semiconductor. The device is also wavelength selective $(2 \mathrm{~nm})$ and operates in the Bragg diffraction regime with only one diffracted beam provided a suitable polarizing setup is used. This technique is very promising for dense optical signal processing provided optical losses of the 1D-PC are lowered, for instance, by using a very thin high-index contrast 1D-PC for the inscription of the transmission grating.

\section{References}

1. E. G. Loewen and E. Popov, Diffraction Gratings and Applications (Dekker, 1997).

2. T. J. Bunning, L. V. Natarajan, V. P. Tondiglia, and R. L. Sutherland, Annu. Rev. Mater. Sci. 30, 83 (2000).

3. J. Zhao, X. Shen, and Y. Xia, Opt. Laser Technol. 33, 23 (2001).

4. Q. Wang, R. M. Brubaker, D. D. Nolte, and M. R. Melloch, J. Opt. Soc. Am. B 9, 1626 (1992).

5. K. M. Kwolek, M. R. Melloch, D. D. Nolte, and G. A. Brost, Appl. Phys. Lett. 67, 736 (1995).

6. L. Menez, I. Zaquine, A. Maruani, and R. Frey, J. Opt. Soc. Am. B 16, 1849 (1999).

7. L. Menez, I. Zaquine, A. Maruani, and R. Frey, Opt. Lett. 27, 479 (2002).

8. A. Moreau, Q. He, I. Zaquine, A. Maruani, and R. Frey, Opt. Lett. 32, 208 (2007).

9. L. Menez, I. Zaquine, A. Maruani, and R. Frey, J. Opt. Soc. Am. B 19, 965 (2002).

10. A. Moreau, I. Zaquine, A. Maruani, and R. Frey, J. Opt. Soc. Am. B 22, 2289 (2005).

11. Q. He, I. Zaquine, A. Maruani, and R. Frey, Opt. Lett. 31, 1184 (2006).

12. R. Frey, Ph. Delaye, and G. Roosen, in Nanophotonics, H. Rigneault, J. M. Lourtioz, C. Delalande, and A. Levenson, eds. (ISTE, 2006), p. 187.

13. Ph. Delaye, M. Astic, R. Frey, and G. Roosen, J. Opt. Soc. Am. B 22, 2494 (2005).

14. D. Bitauld, I. Zaquine, A. Maruani, and R. Frey, Opt. Express 13, 6438 (2005).

15. L. Razzari, D. Träger, M. Astic, P. Delaye, R. Frey, G. Roosen, and R. André, Appl. Phys. Lett. 86, 231106 (2005).

16. R. Pankoke, A. Cassinelli, P. Sillard, C. Dorrer, P. Leisching, and R. Frey, Ann. Phys. 20, 593 (1995).

17. M. Astic, Ph. Delaye, R. Frey, G. Roosen, R. André, N. Belabas, I. Sagnes, and R. Raj (submitted to J. Phys. D). 